\title{
LA-UR-16-22028
}

Approved for public release; distribution is unlimited.

Title: Basic Statistics and Uncertainty Propagation

Author(s): $\quad$ Geist, William H.

Intended for: International Forensics Methodology Training Course

Issued: 2016-03-25 
Disclaimer:

Los Alamos National Laboratory, an affirmative action/equal opportunity employer,is operated by the Los Alamos National Security, LLC for the National NuclearSecurity Administration of the U.S. Department of Energy under contract DE-AC52-06NA25396. By approving this article, the publisher recognizes that the U.S. Government retains nonexclusive, royalty-free license to publish or reproduce the published form of this contribution, or to allow others to do so, for U.S. Government purposes. Los Alamos National Laboratory requests that the publisher identify this article as work performed under the auspices of the U.S. Departmentof Energy. Los Alamos National Laboratory strongly supports academic freedom and a researcher's right to publish; as an institution, however, the Laboratory does not endorse the viewpoint of a publication or guarantee its technical correctness. 


\section{Basic Statistics and Uncertainty Propagation}

Bill Geist

Los Alamos National Laboratory 


\section{Overview}

- Basic Statistical Concepts

- Properties of the Gaussian Distribution

- Error propagation

- Three Example Exercises:

- Uranium Ratios to Determine Potential Source of a Seized Material

- Chronometry to Estimate Age of a Seized Material

- Estimating Amount of a Specified Component 


\section{Uncertainties}

Imagine the results of an isotopic analysis measurements are:

$$
{ }^{234} \mathrm{U}=0.911 \pm 0.034,{ }^{235} \mathrm{U}=92.15 \pm 1.02,{ }^{238} \mathrm{U}=6.08 \pm 0.28
$$

What is the true answer? What do these uncertainties tell us? What do they mean? How can we use them?

To get a better understanding of these results, lets do a thought experiment: What would happen if we repeated the measurement many times?

- The results of each measurement would not be identically the same.

- A histogram of the results would form a Gaussian distribution, centered around the true value*.

- The width of the distribution would be related to the uncertainty.

* Assumes no systematic errors. 


\section{The Gaussian Distribution}

$$
G(x)=\frac{1}{\sigma \sqrt{2 \pi}} e^{-(x-X)^{2} / 2 \sigma^{2}}
$$

$X=$ center of distribution

$=$ average of many measurements

$\sigma=$ width of distribution

= standard deviation after many measurements

$=$ uncertainty determined from counting statistics from one measurement

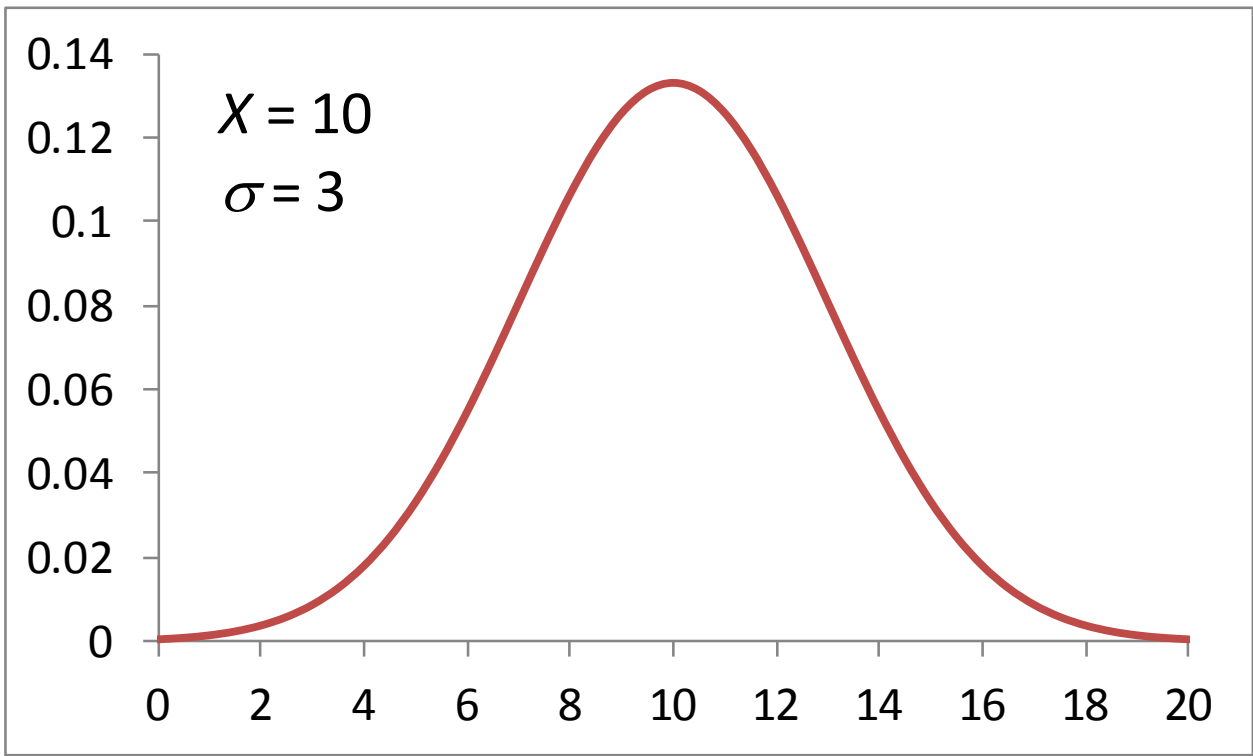

For a large number of measurements, $X$ is a good estimate of the true value. 
Probability within:

$1 \sigma=\int_{-1 \sigma}^{1 \sigma} G(x)=68.3 \%$

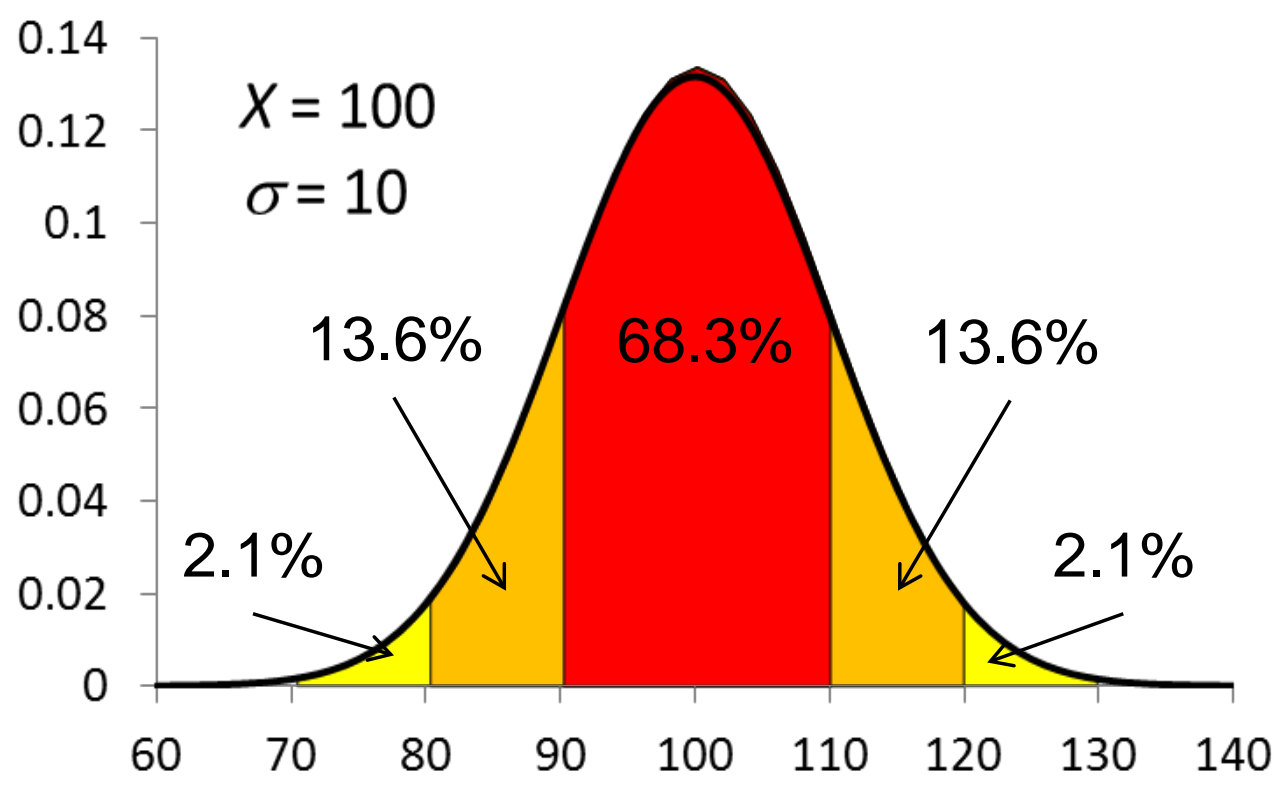

How does this relate if only one measurements is made?

If one measurement is made, there is a $68.3 \%$ chance that the true value is within one $\sigma$ and a $95.5 \%$ chance that the true value is within two $\sigma$. 


\section{Propagation of Error}

The error, $\sigma_{f}$, of a function, $f\left(x_{1}, x_{2}, \cdots, x_{i}\right)$, with $x_{i}$ random

variables is propagated using the follow method:

$$
\sigma_{f}^{2}=\left(\frac{\partial f}{\partial x_{1}} \sigma_{x_{1}}\right)^{2}+\left(\frac{\partial f}{\partial x_{2}} \sigma_{x_{2}}\right)^{2}+\cdots+\left(\frac{\partial f}{\partial x_{i}} \sigma_{x_{i}}\right)^{2}
$$

For example, if $D=A-B$, then $\sigma_{S}=\sqrt{\left(\sigma_{A}\right)^{2}+\left(\sigma_{B}\right)^{2}}$

\begin{tabular}{|c|c|}
\hline$A$ & $12 \pm 0.3$ \\
\hline$B$ & $10 \pm 0.4$ \\
\hline$S$ & $2 \pm 0.5$ \\
\hline$P$ & $120 \pm 5.6$ \\
\hline
\end{tabular}

if $P=A \times B$, then $\sigma_{P}=\sqrt{\left(B \sigma_{A}\right)^{2}+\left(A \sigma_{B}\right)^{2}}$

$$
\begin{aligned}
& \sigma_{P}=\sqrt{\left(A B \frac{\sigma_{A}}{A}\right)^{2}+\left(A B \frac{\sigma_{B}}{B}\right)^{2}} \\
& \sigma_{P}=P \sqrt{\left(\frac{\sigma_{A}}{A}\right)^{2}+\left(\frac{\sigma_{B}}{B}\right)^{2}}
\end{aligned}
$$




\section{Examples}

\section{We will know apply these basic statistical concepts to three examples}

Three Example Exercises:

- Uranium Ratios to Determine Potential Source of a Seized Material

- Chronometry to Estimate Age of a Seized Material

- Estimating Amount of a Specified Component 


\section{Uranium Ratios}

- Suppose that records from Countries A, B, and C indicate the following uranium concentrations and corresponding uncertainties following their most recent processing.

\begin{tabular}{|c|c|c|c|}
\hline Country & Isotope & Weight Percent & $\begin{array}{c}\text { Standard } \\
\text { Uncertainty }\end{array}$ \\
\hline \multirow{2}{*}{$\mathrm{A}$} & $234 \mathrm{U}$ & 0.9449 & 0.0353 \\
& $235 \mathrm{U}$ & 92.8647 & 1.0144 \\
\hline \multirow{2}{*}{$\mathrm{B}$} & $238 \mathrm{U}$ & 5.8159 & 0.2479 \\
\hline & $234 \mathrm{U}$ & 0.9678 & 0.0345 \\
\hline \multirow{2}{*}{$\mathrm{C}$} & $235 \mathrm{U}$ & 91.4354 & 1.0253 \\
& $238 \mathrm{U}$ & 7.2292 & 0.2546 \\
\hline & $234 \mathrm{U}$ & 0.888 & 0.03 \\
\hline
\end{tabular}




\section{Uranium Ratios (cont.)}

- Suppose that a particular quantity of seized material is analyzed and found to have the following uranium concentrations and corresponding uncertainties.

\begin{tabular}{|c|c|c|}
\hline Isotope & $\begin{array}{c}\text { Weight } \\
\text { Percent }\end{array}$ & $\begin{array}{c}\text { Standard } \\
\text { Uncertainty }\end{array}$ \\
\hline $234 \mathrm{U}$ & 0.9113 & 0.0335 \\
$235 \mathrm{U}$ & 92.1501 & 1.0216 \\
$238 \mathrm{U}$ & 6.0753 & 0.2752 \\
\hline
\end{tabular}

- Use this information to determine the most likely country of origin for this seized material. 


\section{Uranium Ratios (cont.)}

- Our strategy will be to compare the ratios

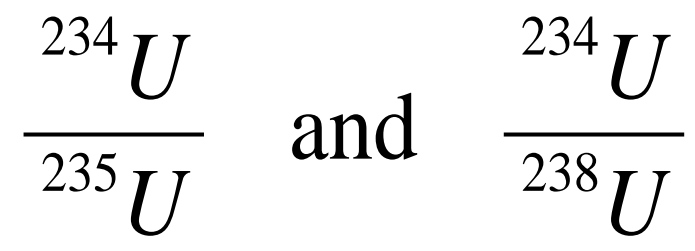

for the seized material versus this same ratios for Countries

$A, B$, and $C$, with an accounting for uncertainty included in the comparison.

- We will then determine whether a specific country of origin can be established with a high level of confidence. 


\section{Uranium Ratios (cont.)}

- These ratios have the functional form

$$
R=\frac{X}{Y}
$$

- Using the propagation of error formulas, we first need the sensitivity coefficients (taking partial derivatives).

$$
\frac{\partial R}{\partial X}=\frac{1}{Y} \quad \text { and } \quad \frac{\partial R}{\partial Y}=\frac{-X}{Y^{2}}
$$

- The general error propagation approximation applied to this function (the ratio), can be expressed and simplified to the following approximation for the variance of $R$ (assuming $X$ and $Y$ are independent).

$$
\hat{\sigma}_{R}^{2} \approx\left(\frac{1}{Y}\right)^{2} \hat{\sigma}_{X}^{2}+\left(\frac{-X}{Y^{2}}\right)^{2} \hat{\sigma}_{Y}^{2}=\left(\frac{X}{Y}\right)^{2}\left[\frac{\hat{\sigma}_{X}^{2}}{X^{2}}+\frac{\hat{\sigma}_{Y}^{2}}{Y^{2}}\right]
$$




\section{Uranium Ratios (cont.)}

- Using the nominal uranium concentrations and corresponding uncertainties listed in the previous tables, the error propagation approximation yields the following values:

\begin{tabular}{|ccc|}
\hline Country & $\begin{array}{c}\text { Ratio } \\
\text { Estimate }\end{array}$ & $\begin{array}{c}\text { Standard } \\
\text { Uncertainty }\end{array}$ \\
\hline A & 0.01018 & 0.00040 \\
0.1625 & 0.0092 \\
\hline B & 0.01058 & 0.00040 \\
C & 0.1339 & 0.0067 \\
\hline \multirow{2}{*}{ Seized Material } & 0.01195 & 0.00043 \\
& 0.1997 & 0.0081 \\
\hline
\end{tabular}




\section{Uranium Ratios (cont.)}

- Note that the resulting standard uncertainties are found by taking a square root of the corresponding variance estimates.

- Applying a multiplier of 2, which represents an approximate 95\% confidence level, to the resulting standard uncertainties yields "expanded uncertainties".

- These expanded uncertainties are depicted in the following figure.

- Based on this figure, it is not likely that the seized material came from Country $\mathrm{C}$.

- We cannot rule out Countries A and B as the potential country of origin for this seized material. 


\section{Uranium Ratios (cont.)}

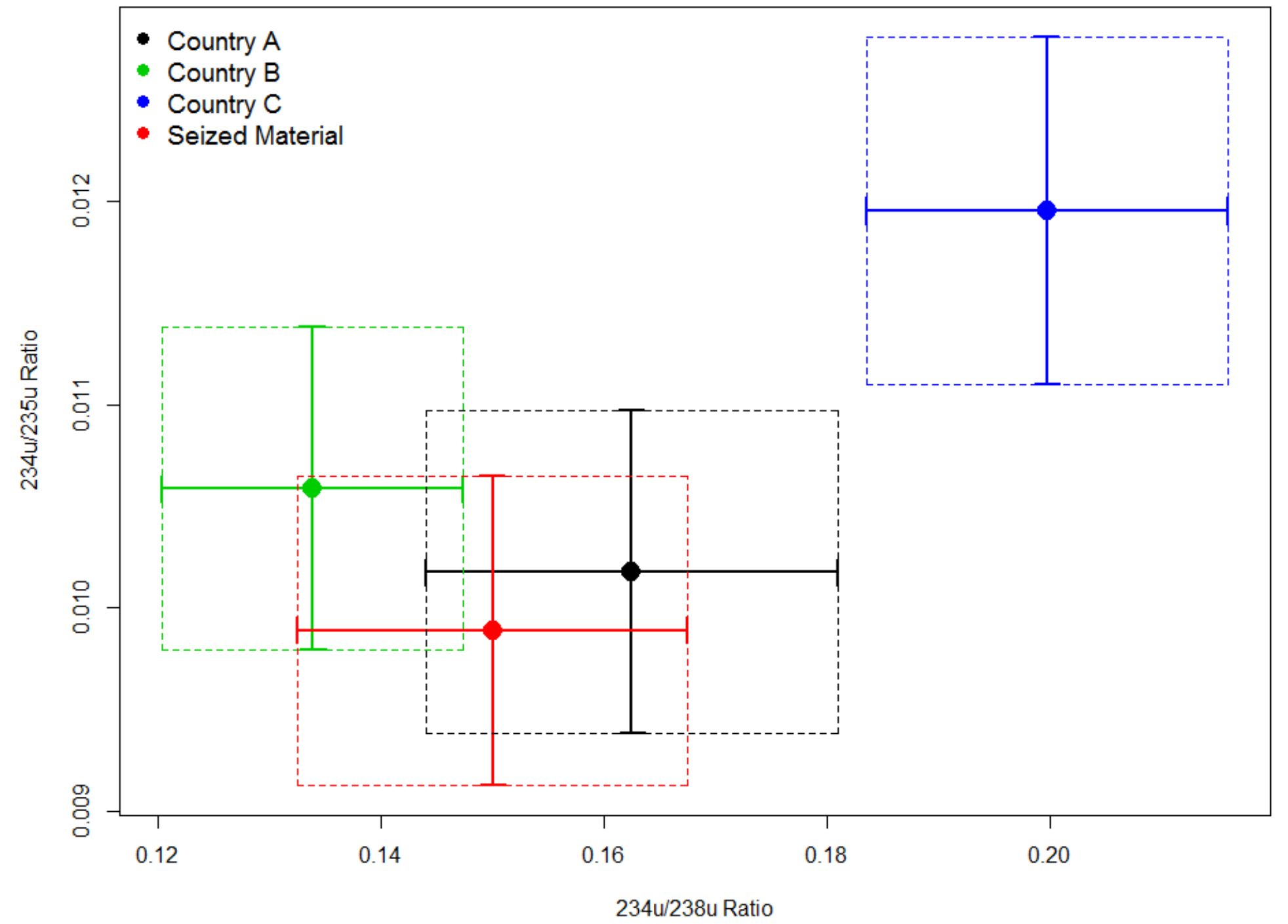




\section{Chronometry for Age Estimation}

- The following formula describes the functional relationship between the proportion of a radioactive isotope at time $t\left(P^{t}\right)$ versus the amount at some starting time $\left(P^{0}\right)$.

$$
P^{t}=P^{0} e^{-\lambda_{P} t}
$$

where $\lambda_{P}=\frac{\ln 2}{t_{1 / 2}}$ and $t_{1 / 2}$ is the half life of the isotope of interest.

- Solving the above equation for $t$ yields

$$
t=\frac{t_{1 / 2} \ln \left(\frac{P^{0}}{P^{t}}\right)}{\ln 2}
$$




\section{Chronometry for Age Estimation (cont.)}

- In the equation for $t$, assume that $P^{0}, P^{t}$, and $t_{1 / 2}$ are considered random variables.

- Therefore, error propagation methods will be used to approximate the uncertainty associated with our estimate of $t$.

- We will need the following partial derivative formulas:

$\frac{\partial t}{\partial P^{0}}=\frac{t_{1 / 2}}{P^{0} \ln 2}, \quad \frac{\partial t}{\partial P^{t}}=\frac{-t_{1 / 2}}{P^{t} \ln 2}, \quad$ and $\quad \frac{\partial t}{\partial t_{1 / 2}}=\frac{1}{\ln 2} \ln \left(\frac{P^{0}}{P^{t}}\right)$ 


\section{Chronometry for Age Estimation (cont.)}

- Error propagation methodology yields the following formula for approximating the variance associated with our estimate of $t$ (assuming $P^{0}, P^{t}$, and $t_{1 / 2}$ are independent).

$$
\hat{\sigma}_{t}^{2} \approx\left(\frac{t_{\frac{1}{2}} \ln \left(\frac{P^{0}}{P^{t}}\right)}{\ln 2}\right)^{2}\left[\frac{\hat{\sigma}_{t_{0.5}}^{2}}{t_{1 / 2}^{2}}+\frac{\hat{\sigma}_{P^{0}}^{2}}{\left(P^{0}\right)^{2}\left[\ln \left(\frac{P^{0}}{P^{t}}\right)\right]^{2}}+\frac{\hat{\sigma}_{P^{t}}^{2}}{\left(P^{t}\right)^{2}\left[\ln \left(\frac{P^{0}}{P^{t}}\right)\right]^{2}}\right]
$$




\section{Chronometry for Age Estimation (cont.)}

- Suppose a particular quantity of seized material contains some proportion of ${ }^{241} \mathrm{Am}$ as well as ${ }^{241} \mathrm{Pu}$, as described in the table below. Assume a half-life of 14 years for ${ }^{241} \mathrm{Pu}$.

\begin{tabular}{|c|c|c|}
\hline $\begin{array}{c}\text { Random } \\
\text { Variable }\end{array}$ & Nominal Value & $\begin{array}{c}\text { Standard } \\
\text { Uncertainty }\end{array}$ \\
\hline$t_{1} / 2$ & 14 & 0.001 \\
\hline${ }^{241} \mathrm{Pu}: P^{0}$ & 1 & 0.005 \\
\hline${ }^{241} \mathrm{Pu}: P^{t}$ & 0.9317 & 0.004 \\
\hline
\end{tabular}

- Use this information to estimate the age of the seized material (time since last processing/separation).

- Calculate an approximation for the standard uncertainty associated with your estimate of the material's age. 
Chronometry for Age Estimation

- $t=1.43$ years (the estimated age of the material, the time since the material was last processed).

- The standard uncertainty associated with our estimate of $t$ is $\sigma_{t}=0.13$ years. This is the square root of the variance estimate.

- Therefore, there is a

- $69 \%$ confidence that the true age is $1.43 \pm 0.13$ years

- $95 \%$ confidence that the true age is $1.43 \pm 0.26$ years

- $99.7 \%$ confidence that the true age is $1.43 \pm 0.39$ years 


\section{Estimating the Amount of a Specified Component}

- Suppose that the measured total mass of a particular seized material is $M$.

- A sample of the material is taken to be analyzed. The measured mass of the sample is $m$.

- The analyzed amount of the component of interest in the sample is $x$.

- Estimate the amount $X$ of the specified component in the entire quantity of the seized material, and the associated standard uncertainty. 


\section{Estimating the Amount of a Specified Component (cont.)}

- Assuming the proportion of the specified component is the same for both the entire quantity of seized material and the analyzed sample,

$$
\frac{X}{M}=\frac{x}{m}
$$

- Solving this equation for $X$ yields:

$$
X=M \frac{X}{m}
$$




\section{Estimating the Amount of a Specified Component (cont.)}

- Assume that $M, x$, and $m$ are random variables with nominal values and associated uncertainties given in the table below.

\begin{tabular}{|cccc|}
\hline $\begin{array}{c}\text { Random } \\
\text { Variable }\end{array}$ & $\begin{array}{c}\text { Nominal } \\
\text { Value }\end{array}$ & $\begin{array}{c}\text { Relative } \\
\text { Standard } \\
\text { Uncertainty }\end{array}$ & $\begin{array}{c}\text { Standard } \\
\text { Uncertainty }\end{array}$ \\
\hline$M$ & $2450 \mathrm{~g}$ & $1 \%$ & $24.50 \mathrm{~g}$ \\
$x$ & $4 \mathrm{~g}$ & $3 \%$ & $0.12 \mathrm{~g}$ \\
$m$ & $210 \mathrm{~g}$ & $1 \%$ & $2.10 \mathrm{~g}$ \\
\hline
\end{tabular}

- We will need the following partial derivatives:

$$
\frac{\partial X}{\partial M}=\frac{x}{m}, \quad \frac{\partial X}{\partial x}=\frac{M}{m}, \quad \text { and } \quad \frac{\partial X}{\partial m}=\frac{-M x}{m^{2}}
$$




\section{Estimating the Amount of a Specified Component (cont.)}

- Error propagation methodology yields the following formula for approximating the variance associated with our estimate of $X$ (assuming $M, x$, and $m$ are independent).

$$
\hat{\sigma}_{X}^{2} \approx\left(\frac{M x}{m}\right)^{2}\left[\frac{\hat{\sigma}_{M}^{2}}{M^{2}}+\frac{\hat{\sigma}_{x}^{2}}{x^{2}}+\frac{\hat{\sigma}_{m}^{2}}{m^{2}}\right]
$$




\section{Estimating the Amount of a Specified Component (cont.)}

- The assumed inputs yield the following estimates:

- $X=46.7 \mathrm{~g}$.

- The standard uncertainty associated with our estimate of $X$ is $\sigma_{X}=1.6 \mathrm{~g}$. This is the square root of the variance estimate.

- Therefore, there is a

- $69 \%$ confidence that the true mass is $46.7 \pm 1.6$ grams

- $95 \%$ confidence that the true mass is $46.7 \pm 3.2$ grams

- $99.7 \%$ confidence that the true mass is $46.7 \pm 4.8$ grams 


\section{Summary}

Understanding the uncertainties is critical to draw conclusions about a measurement.

When performing calculations of measured quantities, one must correctly propagate the errors to get the uncertainty of the calculated quantity.

A $1 \sigma$ uncertainty will result in a confidence level of $68.3 \%$ A $2 \sigma$ uncertainty will result in a confidence level of $95.5 \%$ A $3 \sigma$ uncertainty will result in a confidence level of $>99 \%$ 